\title{
The Mediator complex subunits MED25/PFT1 and MED8 are required for transcriptional responses to changes in cell wall arabinose composition and glucose treatment in Arabidopsis thaliana
}

\author{
Mathilde Seguela-Arnaud ${ }^{1,2}$, Caroline Smith ${ }^{1}$, Marcos Castellanos Uribe ${ }^{3}$, Sean May ${ }^{3}$, Harry Fischl 1,4,
}

Neil McKenzie ${ }^{1}$ and Michael W. Bevan ${ }^{1 *}$ (D)

\begin{abstract}
Background: Plant cell walls are dynamic structures involved in all aspects of plant growth, environmental interactions and defense responses, and are the most abundant renewable source of carbon-containing polymers on the planet. To balance rigidity and extensibility, the composition and integrity of cell wall components need to be tightly regulated, for example during cell elongation.

Results: We show that mutations in the MED25/PFT1 and MED8 subunits of the Mediator transcription complex suppressed the sugar-hypersensitive hypocotyl elongation phenotype of the hsr8-1 mutant, which has cell wall defects due to arabinose deficiency that do not permit normal cell elongation. This suppression occurred independently of light and jasmonic acid (JA) signaling. Gene expression analyses revealed that the expression of genes induced in hsr8-1 that encode enzymes and proteins that are involved in cell expansion and cell wall strengthening is reduced in the pft1-2 mutant line, and the expression of genes encoding transcription factors involved in reducing hypocotyl cell elongation, genes encoding cell wall associated enzymes and proteins is up-regulated in pft1-2. PFT1 was also required for the expression of several glucose-induced genes, including those encoding cell wall components and enzymes, regulatory and enzymatic components of anthocyanin biosynthesis, and flavonoid and glucosinolate biosynthetic pathways.
\end{abstract}

Conclusions: These results establish that MED25 and MED8 subunits of the Mediator transcriptional complex are required for the transcriptional regulation of genes involved in cell elongation and cell wall composition in response to defective cell walls and in sugar- responsive gene expression.

\section{Background}

Sugars are universal nutrients that provide carbon skeletons for energy production, storage and the synthesis of most metabolites. In plants, the main sink of carbon is the cell wall [1], a dynamic structure that provides both rigidity to support the plant and plasticity to allow cell growth. There is extensive knowledge of the enzymes involved in the synthesis and assembly of cell wall polysaccharides [2-4], but relatively little is known about how environmental stimuli and photosynthate availability contribute to cell wall formation during cell growth.

\footnotetext{
* Correspondence: michael.bevan@jic.ac.uk

${ }^{1}$ Cell and Developmental Biology Department, John Innes Centre, Colney Lane, Norwich NR4 7UH, UK

Full list of author information is available at the end of the article
}

Sugars can act as both metabolic intermediates and as signaling molecules [5], and treatment of plants with sugars promotes growth. One mechanism linking sugar availability and growth promotion is the stimulation of auxin synthesis by exogenous sugars [6], which may indirectly influence cell wall formation by promoting cell elongation. Sugar levels may also link cell wall formation with the maintenance of turgor pressure. Mutations in a gene encoding a cell wall-associated kinase (WAK), which is required for normal cell expansion, also exhibited reduced vacuolar invertase activity [7]. This led to an increased dependence of seedlings on exogenous sugars for maintaining turgor and growth, and indicated that WAKs may be involved in maintaining the balance between turgor pressure, which drives cell expansion, and 
cell wall formation. A similar link between turgor and cell walls was shown by interrupting cellulose synthesis and observing that the resulting stress responses and distorted cells were rescued by osmotic support and sugar availability [8]. The interaction between sugar signaling and cell wall integrity control was also highlighted by the sugar hypersensitivity of several cell wall matrix structural mutants mur4, mur1 and mur3 [9]. The hsr8-1 (high sugar response 8-1) allele of MUR4, which is defective in UDPArabinose synthesis, exhibits sugar hypersensitive gene expression and growth responses [9]. The pleitropic regulatory locus1 (prl1) mutation was identified as a suppressor of hsr8-1 sugar hypersensitivity phenotypes. PRL1 (Pleiotropic Regulatory Locus 1) encodes a WD40 protein that is a component of a spliceosome complex, and prl1 mutations have multiple complex phenotypes that include sugar hypersensitivity [10]. These findings suggest that impaired cell wall composition may be actively sensed, leading to transcriptional responses that modify cell wall composition and growth [11].

Recently, the existence of such transcriptional regulators controlling cell wall integrity and plant growth was demonstrated $[12,13]$. The stunted growth and lignin deficiency of the lignin deficient mutant ref8 was restored by the disruption of two subunits of the transcriptional regulatory complex Mediator, MED5a and MED5b. Here we show that the MED25/PFT1 (MEDIATOR25/PHYTOCHROME AND FLOWERING TIME 1) and MED8, two other subunits of the Mediator transcription complex, are able to suppress the sugar hypersensitive short hypocotyl and gene expression phenotypes of the hsr8-1 mutant. We show that these Mediator subunits are required for the altered expression of a set of genes encoding cell wall components and biosynthetic activities in the hsr8-1 mutant [9]. We show that one of these subunits, MED25/PFT1, is also required for the coordinated induction of several sugar-responsive genes, including those encoding cell wall modifying enzymes. These results suggest the MED25 and MED8 subunits of the Mediator complex have an integrating role by linking sugar responsive- and cell wall- gene expression.

\section{Results}

\section{Identification of a novel suppressor of $h s r 8-1$ sugar} hypersensitive growth

The high sugar response8-1 mutant, which has reduced cell wall arabinose [14], displays a range of sugar hypersensitivity phenotypes [9]. Among these, dark grown hsr8-1 seedlings show reduced hypocotyl elongation in response to glucose in comparison to wild-type plants, and light-grown seedlings show hypersensitive sugarregulated gene expression and anthocyanin content. To identify possible mechanisms linking altered cell wall composition and sugar responses, we screened for suppressors of the short hypocotyl phenotype of the hsr8-1 mutant. We grew M2 seedlings of a fast neutron mutagenized $h s r 8-1$ population in the dark in the presence of glucose for 14 days and screened for individuals with longer hypocotyls. Eight suppressors of hsr8-1 (soh) were isolated, several deletions were genetically mapped, and the soh715hsr8-1 recessive mutant was selected for further analysis. Figures $1 \mathrm{a}$ and $\mathrm{b}$ show the intermediate
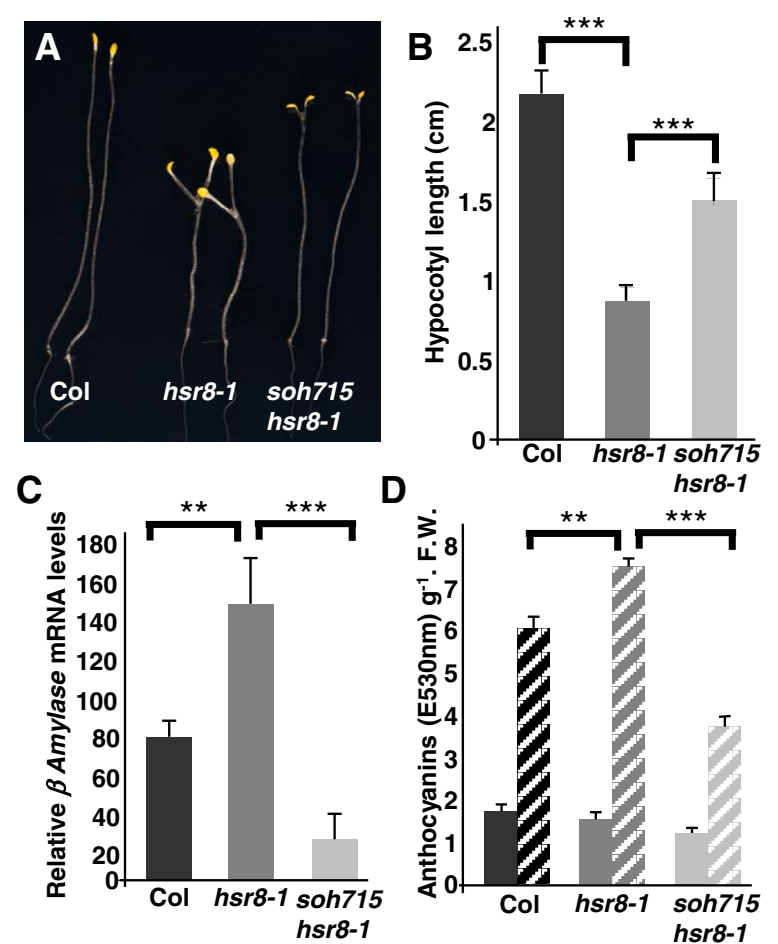

Fig. 1 Identification of a suppressor of hsr8-1 sugar-hypersensitive hypocotyl elongation in the dark. a Image of sugar hypersensitive hypocotyl elongation of Col, hsr8-1 and hsr8-1soh715 grown on 1 \% glucose on vertical plates in the dark. $\mathbf{b}$ Quantitative measurements of hypocotyl lengths of Col, hsr8-1 and hsr8-1soh715. Seedlings were grown vertically in the dark for 14 days on MS medium with $1 \%$ Glucose. Errors bars represent SD $(n>30)$. ${ }^{* * *}, p<0.001$ comparing Col to hsr8-1 and hsr8-1 to soh715 hsr8-1 (Student's t- test). Data shown is representative of three independent experiments. c Quantitative Real-time PCR analysis of $\beta$-Amylase mRNA levels in Col, hsr8-1 and the hsr8-1soh715 repressor in response to glucose. Seedlings were grown on MS medium supplemented with $0.5 \%$ glucose in constant light. After 7 days, seedlings were transferred $24 \mathrm{~h}$ in a MS glucose-free liquid medium and then treated for $6 \mathrm{~h}$ with $3 \% \mathrm{MS}$ medium containing $3 \%$ glucose. Errors bars represent SD from three biological replicates. Data shown is representative of three independent experiments. ${ }^{*}, p<0.01$ comparing Col to hsr8-1; ***, $p<0.001$ comparing hsr8-1 to soh715 hsr8-1 (Student's t- test). Relative transcript levels (RTL) were calculated using transcript levels of the reference gene TUB6 (At5g12250). d Anthocyanin accumulation in response to glucose in Col, hsr8-1 and hsr8-1soh715. Seedlings were grown in continuous light for 7 days on MS medium containing $1 \%$ glucose (solid bars) or $3 \%$ glucose (dashed bars). Errors bars represent SD from three biological replicates. ${ }^{* *}, p<0.01$ comparing Col to hsr8-1; ${ }^{* * *}, p<0.001$ comparing hsr8-1 to soh715 hsr8-1 (Student's t- test). Data shown is representative of two independent experiments 
hypocotyl length of the soh715hsr8-1 suppressor mutant compared to wild- type $\mathrm{Col}$ and $h s r 8-1$. The hsr8-1 sugar hypersensitive phenotypes, increased $\beta$-amylase $(B A M)$ mRNA accumulation and anthocyanin content were also suppressed in the soh715hsr8-1 mutant, with BAM mRNA accumulation and anthocyanin content reduced in hsr8-1 to lower levels than in wild-type plants (Fig. 1c and d). These results show that the soh715 mutation suppresses hsr8-1 sugar hypersensitive phenotypes.

\section{soh715 is allelic to the pft1-2 mutation}

To map the soh715 locus in the Columbia ecotype, the double mutant was crossed with wild-type Landsberg erecta. To isolate soh715hsr $8-1$ double mutant plants, long hypocotyl plants were selected in the F2 population and genotyped to identify hsr8-1 homozygous plants. Double soh715hsr8-1 mutants comprised $1 / 4^{\text {th }}$ of the segregating population instead of the expected $1 / 16^{\text {th }}$. Preliminary genetic analysis (data not shown) showed the soh715 locus mapped to a region of chromosome 1 where HSR8 also maps, confirming that the soh715 and hsr8-1 mutations may be genetically linked. A transcriptbased cloning approach [15] was then used to identify deletions in the mapped region. Gene expression in hsr8-1 and soh175hsr8-1 seedlings was assessed using the ATH1 Gene Chip. Comparison of gene expression levels revealed that 6 consecutive genes on chromosome 1 showed strongly reduced RNA levels in soh715hsr8-1 compared to hsr8-1 (At1g25510, At1g25520, At1g25530, At1g25540, At1g25550, At1g25560; Fig. 2a). This result,
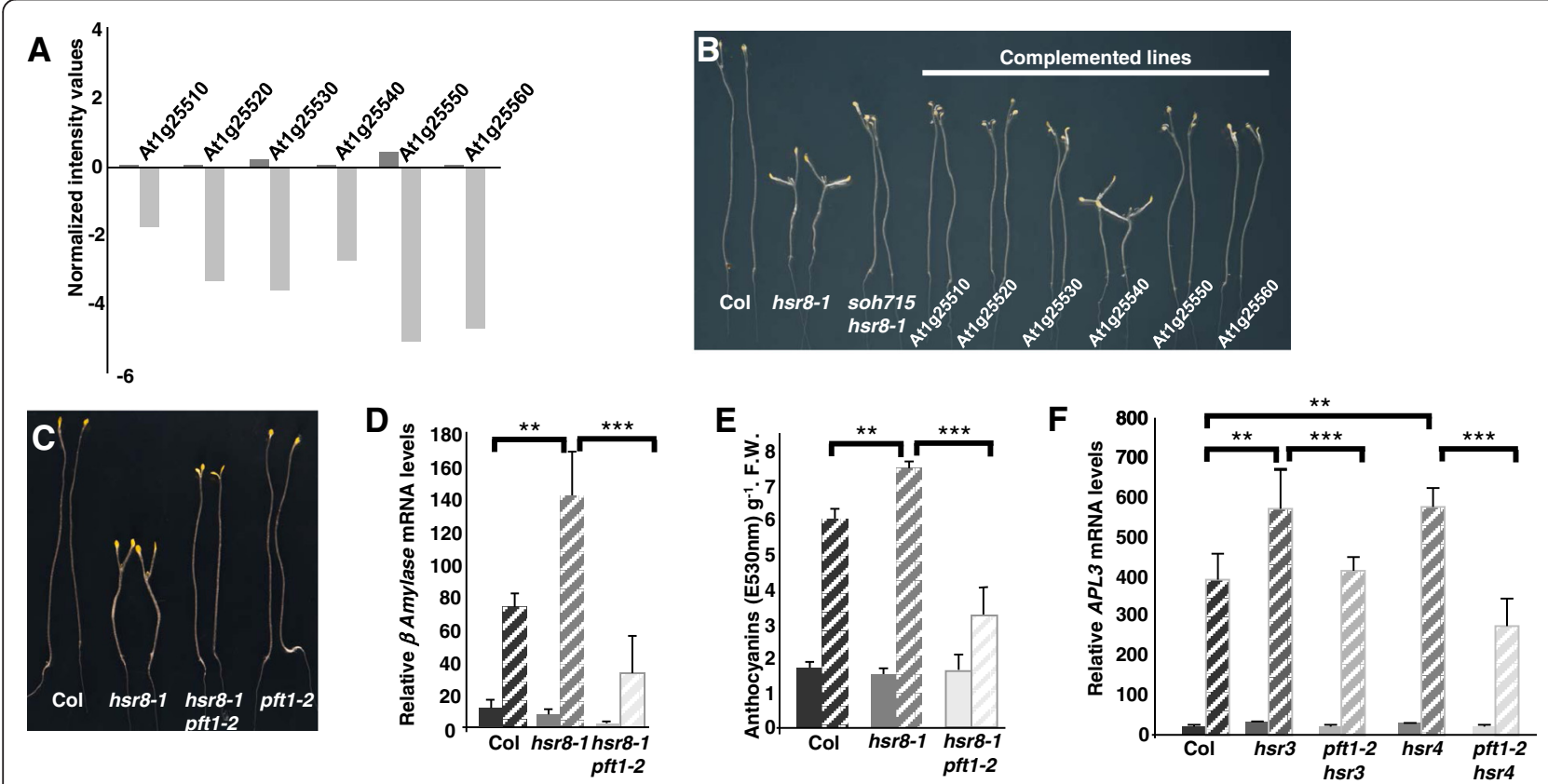

Fig. 2 hsr8-1 sugar hypersensitive phenotypes are suppressed by the pft1-2 mutation. a Identification by microarray analysis of a cluster of six genes that are down regulated in the suppressor line soh715hsr8-1 compared to hsr8-1. Values on the Y-axis are those obtained after normalization of the entire microarray data set. Dark grey bars and light grey bars represent values obtained for the hsr8-1 mutant and the hsr8-15oh715 suppressor line respectively. b Sugar hypersensitive dark development of Col, hsr8-1, soh715hsr8-1 and soh715hsr8-1 complemented with each of the 6 genes of the deletion. Seedlings were grown vertically in the dark for 14 days on MS medium containing $1 \%$ Glucose. Only the genomic fragment containing the At1g25540 gene rescued the dark development phenotype. c Sugar hypersensitive dark development of Col, hsr8-1, the double mutant hsr8-1pft1-2 and pft1-2. Seedlings were grown as described in (B) above. $\mathbf{d}$ Quantitative Real-time PCR analysis of $\beta$-Amylase mRNA levels in Col, hsr8-1 and the double mutant hsr8-1pft1-2 in response to glucose. Seedlings were grown on MS medium supplemented with $0.5 \%$ glucose in constant light. After 7 days, the seedlings were transferred for $24 \mathrm{~h}$ to MS glucose-free liquid medium and then treated for $6 \mathrm{~h}$ with MS medium containing $3 \%$ glucose. Errors bars represent SD from three biological replicates. Data shown is representative of three independent experiments. ${ }^{*}, p<0.01 \mathrm{comparing}$ Col to hsr8-1; ***, $p<0.001$ comparing hsr8-1 to hsr8-1 pft1-2 (Student's t- test). Relative transcript levels (RTL) were calculated using transcript levels of the reference gene TUB6 (At5g12250). e Anthocyanin accumulation in response to glucose in Col, hsr8-1 and the double mutant hsr8-1 pft 1-2. Seedlings were grown in continuous light for 7 days on MS medium containing $1 \%$ glucose (solid bars) or $3 \%$ glucose (dashed bars). Errors bars represent SD from three biological replicates. Data shown is representative of two independent experiments. ${ }^{* *}, p<0.01$ comparing Col to hsr8-1; ${ }^{* * *}, p<0.001$ comparing hsr8-1 to hsr8-1 pft1-1 (Student's t- test). f Quantitative Real-time PCR analysis of the sugar-responsive APL3 gene mRNA levels in Col, hsr3, pft1-2hsr3, hsr4, pft1-2hsr4 and pft1-2 in response to glucose. Hsr3 and hsr4 are sugar-hypersensitive mutations in subunits of the ARP2/3 complex [18]. Seedlings were grown on MS medium supplemented with $0.5 \%$ glucose in constant light. After 7 days, the seedlings were transferred to glucose-free liquid MS medium for $24 \mathrm{~h}$ and then treated for $6 \mathrm{~h}$ with MS medium containing either $0 \%$ glucose (solid bars) or $3 \%$ glucose (dashed bars). Errors bars represent SD from three biological replicates. ${ }^{* *}, p<0.01$ comparing Col to $h s r 3$ and Col to hsr4; ${ }^{* * *}, p<0.001$ comparing hsr3 to hsr3 pft1-2 and hsr4 to hsr4 pft1-2 (Student's t- test). Relative transcript levels (RTL) were calculated using transcript levels of the reference gene TUB6 (At5g12250) 
taken together with the preliminary genetic mapping data, indicated that a deletion encompassing 6 genes on chromosome 1 suppressed the hsr8-1 phenotype. To identify the gene(s) involved, we complemented the soh715hsr8-1 mutant background with 6 genomic fragments, each containing one gene and flanking regions in the deleted locus. Only the genomic fragment containing At1g25540 restored the hsr8-1 short hypocotyl phenotype (Fig. 2b). The suppression of hsr8-1 dark development phenotype in the soh715hsr8-1 mutant is therefore caused by the deletion of At1g25540, encoding the MED25/PFT1 protein [16, 17]. To confirm this observation a double mutant between $h s r 8-1$ and a loss-of function T-DNA insertion allele in At1g25540 called pft1-2 was analysed. When grown in the dark in the presence of glucose, the hsr8-1pft2-1 double mutant displayed the same intermediate hypocotyl length as soh715hsr8-1 (Fig. 2c). Increased accumulation of $B A M$ transcripts and anthocyanins in $h s r 8-1$ in response to glucose treatment was suppressed by the pft1-2 mutation (Fig. $2 \mathrm{~d}$ and e). Figure $2 \mathrm{f}$ shows that $p f t 1-2$ also suppresses elevated glucose- responsive APL3 expression in the glucose hypersensitive mutants hsr3 [18] and $h s r 4$, which is a mis-sense mutation in the ARP3 subunit of the Arp $2 / 3$ complex(unpublished data). Therefore loss of PFT1 gene function suppressed the hsr8-1 hypocotyl cell elongation defect and sugar hypersensitive gene expression.

\section{Cell wall composition is altered in pft1-2}

Figure 3a shows that reduced hypocotyl elongation in hsr $8-1$, and its suppression by pft1-2, was due to changes in cell length and not in cell number. The suppression of the short hypocotyl phenotype in hsr8-1 by pft1-2 was not due to changes in cell wall monosaccharide composition, as the hsr8-1pft1-2 double mutant had the same reduced

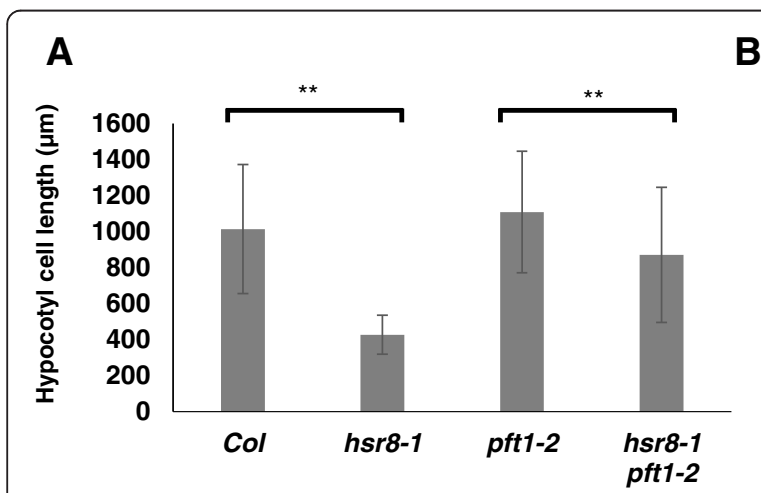

C

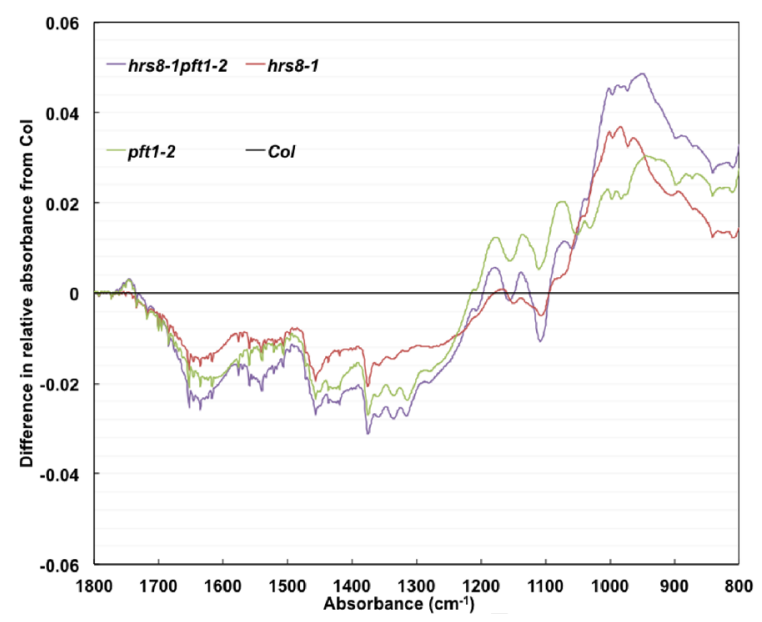

B

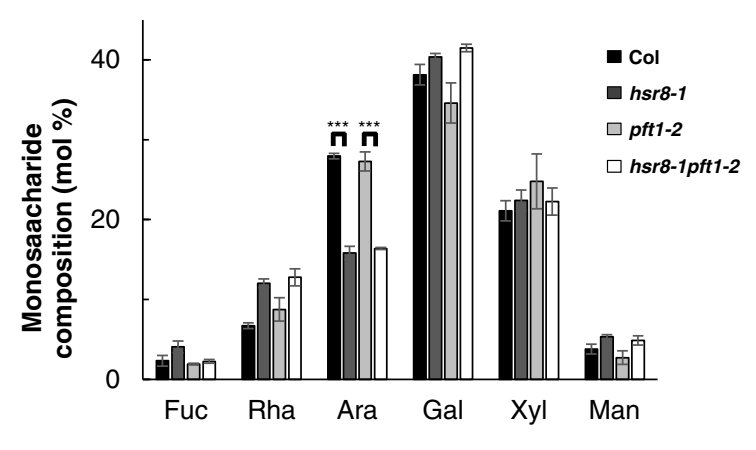

D

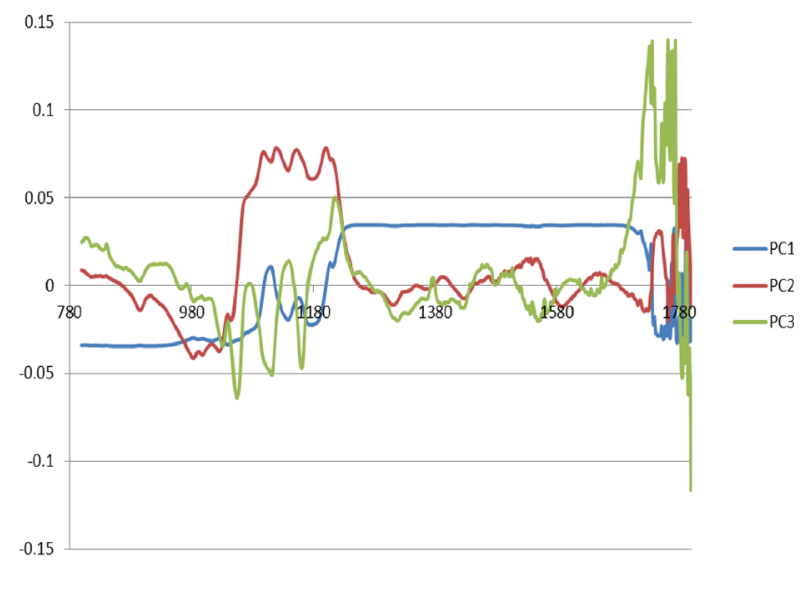

Fig. 3 Comparison of cell elongation and cell wall composition in Col, hsr8-1, pft1-2 and hsr8-1pft1-2. a Hypocotyl cell length was measured from scanning electron micrograph images. $n=10$ cells each from 5 hypocotyls. ${ }^{*}, p<0.01$ comparing hsr8-1 to Col, and hsr8-1 to pft 1-2 and hsr8-1 pft1-2 (Student's t- test). b Monosaccharide composition of cell wall material isolated from 14 day old light grown seedlings (5 biological replicates). Fuc fucose; Rha rhamnose; Ara arabinose; Xyl xylose; Man mannose. . ***, $p<0.001$ comparing arabinose levels in Col to hsr8-1, and pft1-2 to hsr8-1 pft1-2 (Student's t- test). c Compositional analysis of cell wall material isolated from dark grown hypocotyl tissues using FTIR. The data are represented as differences in relative absorbance from wild-type Col. $\mathbf{d}$ Principal Components Analyses of FTIR data. Score loadings of PC1, PC2 and PC3 are plotted against the range of wavelengths to show the major variance 
levels of arabinose as hsr8-1 (Fig. 3b). Additional analyses of cell wall composition of hypocotyls of dark-grown Col, hsr8-1, hsr8-1pft1-2 and pft1-2 mutants seedlings were conducted using Fourier Transform InfraRed spectroscopy (FTIR) [19]. Figure 3c shows difference spectra relative to wild-type Col, and Principle Components Analysis (PCA) identified three principle components when mapped as score loadings (Fig. 3d). PC1 explained $\sim 80 \%$ of the variation in cell wall composition between genotypes, showing very broad variation across the spectra with positive loadings between 800 and $1200 \mathrm{~cm}-1$ and depletion at 1200$1800 \mathrm{~cm}-1$ relative to Columbia. Although PC2 and PC3 explained less variation ( 15 and $4 \%$ respectively), these principle components identified variation in more specific spectra between genotypes. In PC2, the positive loading between 1120 and $1097 \mathrm{~cm}-1$ may reflect variation in xyloglucan and pectin respectively between genotypes [19]. PC3 identifies positive loadings between 1660 and $1776 \mathrm{~cm}-1$, possibly reflecting differences in waxes and phenolic composition [19]. Additional file 1: Figure S1 are scatter plots comparing PC1, PC2 and PC2 between the mutants. There were significant differences between each of the genotypes for each of the three PCs.

\section{Suppression of $h s r 8-1$ by pft 1-2 is not dependent on the phyA, phyB or jasmonate pathways}

MED25/PFT1 was first identified as a positive regulator of flowering in response to sub-optimal light conditions, and pft 1 mutants display slightly longer hypocotyls in far red light and a late flowering phenotype in long days [16]. As mutants with longer hypocotyls were identified in our screen, and because phyA has been implicated in sugar responses [20], we assessed the role of phytochrome signalling pathways in the suppression of $h s r 8-1$ sugar hypersensitivity. Neither the phyA-201 [21] nor the phyB-1 [22] mutations suppressed the hsr8-1 dark development phenotype (Fig. 4a). Seedlings were also grown under constant white light (Fig. 4b) and constant far-red light (Fig. 4c) to confirm that the phyA-201hsr8-1 and $p h y B-1 h s r 8-1$ double mutants displayed characteristic $p h y A$ and $p h y B$ phenotypes, unlike the $p f t 1-2 h s r 8-1$ double mutant.

PFT1 is a regulator of the jasmonate (JA) signalling pathway [23]. As cell wall defects can trigger defence responses through the jasmonate signalling pathway [24, 25], we tested whether JA-dependent defence responses were activated in $h s r 8-1$ and if $p f t 1-2$ suppressed $h s r 8-1$ sugar hypersensitivity through the JA signalling pathway. Expression of $V S P 1, V S P 2$ and ERF1, which are strongly up regulated by JA, was not up- regulated in hsr8-1 compared to Col in dark-grown seedlings (Additional file 1: Figure S2). This showed that the JA pathway was not induced in hsr8-1 in response to its cell wall defect. Crosses to the JA- insensitive mutant coi1-16 [26] confirmed this; the coil-

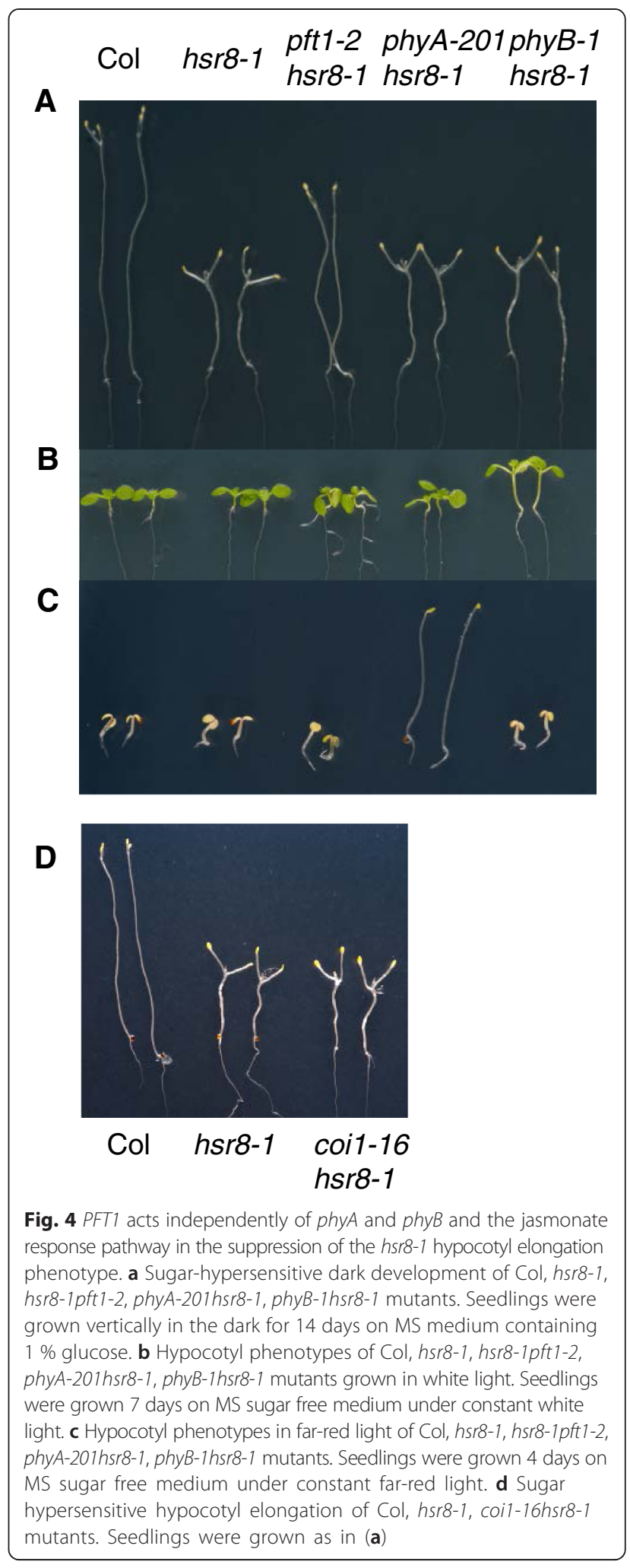

16hsr8-1 double mutant had the same short hypocotyl phenotype as hsr8-1 (Fig. 4d). Therefore suppression of the hsr8-1 short hypocotyl phenotype by pft1-2 is 
independent of its role in the JA and phytochrome signalling pathways.

\section{Microarray analysis of pft1-2-dependent gene expression} PFT1 encodes subunit 25 of the Mediator complex, a conserved regulator of transcription in eukaryotes [17, 27]. We therefore assessed the extent to which PFT1 controls gene expression in response to glucose in light grown seedlings, and also how it controls gene expression during dark development in Col and hsr8-1 genetic backgrounds. For glucose-responsive gene expression, three independent replicates of $p f t 1-2$ and wild-type light-grown 7 day old seedlings were collected $6 \mathrm{~h}$ after $3 \%$ glucose or $0 \%$ glucose treatment. Two-way ANOVA (Analysis of Variance) (Additional file 2) revealed that 1438 genes were differentially expressed in response to $3 \%$ glucose in Col and 1346 genes in pft1-2 (Fig. 5a), of which 931 genes were differentially expressed in response to glucose in both genotypes. A total of 92 genes had fold changes between -2 and +2 , and 47 genes were induced $>2$ fold by glucose in wild-type Col. Nineteen of these showed no significant glucose- dependent induction in pft1-2 and 28 showed strongly reduced glucose- dependent induction in pft1-2 (Fig. 5b and c). The expression of five general categories of genes were either completely or partially dependent on PFT1 for increased expression in response to glucose. Expression of six genes involved in the regulation, biosynthesis and transport of anthocyanins required $P F T 1$, including the central regulator $M Y B 75 / P A P 1$ [28]. Five genes encoding uptake transporters of nitrate, phosphate and sulphate, and the phosphate uptake regulator SPX3, also required PFT1 for increased expression in response to glucose $[29,30]$. Seven genes encoding enzymes (primarily cytochrome P540s) in the biosynthesis of glucosinolates required PFT1 for their expression in response to glucose. Of the four MYB transcription factor genes involved in regulating glucosinolate (GSL) biosynthesis [31, 32], MYB28/HAG1 required PFT1 for increased expression. Thirteen genes encoding a wide variety of stress responsive genes required PFT1 for expression in response to glucose. These include two COR (COld Regulated)-related genes, NCED3 involved in ABA biosynthesis, the AFP1 gene encoding an ABI5 binding protein, the heat-shock transcription factor $H S F A 2$, a PIRIN gene involved in ABA signaling, bZIP44 involved in regulating proline dehydrogenase, and ECA2 encoding an ER $\mathrm{Ca}^{2+}$ transporter involved in stress responses. Finally, several genes encoding proteins involved in cell expansion required PFT1 for their glucose-responsive expression, including two Lipid Transfer Proteins (LTPs) involved in membrane modifications, and
Expansin 4, which is involved in cell wall extension $[33,34]$.

To confirm and extend these microarray analyses, we measured the influence of PFT1 on the expression of a small set of well- characterised glucose-responsive genes identified previously in microarray experiments [35]. QRTPCR analysis showed that the glucose-induced genes APL3, BAM, GBSS1, GPT2 and PDC1 all had reduced expression in $p f t 1-2$ (Additional file 1: Figure S3A-S3E), and confirmed the microarray data showing reduced expression of $A P L 3$ and BAM. The expression of genes encoding enzymes in the anthocyanin synthesis pathway (FLS, CHS and TT6) was also assessed by Q-RTPCR, and they all showed reduced expression in pft1-2 (Additional file 1: Figure S3F-3H). Finally, anthocyanin levels were reduced to $45 \%$ of wild-type levels in pft1-2 (Additional file 1: Figure S2I), confirming the important role of PFT1 in the expression of regulators and enzymes of anthocyanin synthesis.

Gene expression in 14-day old dark-grown seedlings of Col, hsr8-1, hsr8-1pft1-2 and pft1-2 was measured in three independent RNA samples using microarray analysis. Two-way ANOVA analysis (Additional file 3) identified 76 genes that were $\geq 2$ fold up- or down- regulated in $h s r 8-1$ compared to Col, and 44 genes were differently regulated between $h s r 8-1$ and $h s r 8-1 p f t 1-2$. There were 29 genes in common that were differentially regulated in $h s r 8-1$ vs Col and $h s r 8-1$ vs hsr8-1pft1-2. These genes were clustered according to their expression patterns (Fig. 5d and e). Of the 15 genes that were significantly down- regulated in hsr8-1 compared to Col and up- regulated in pft1-2hsr8-1 compared to hsr8-1 (that is, requiring PFT1 for repressing their expression in hsr8-1), 10 encode proteins involved in cell wall formation, cuticle formation and cell expansion. These include XTH17 and XTH20, encoding xyloglucan endotransglycosidase/hydrolase enzymes that cleave and rearrange xyloglucans [36]), and IRX9 encodes a xylosyl transferase involved in xylan synthesis [37]. EXPB5 and EXP5 encode the cell wall proteins expansin B3 and expansin 5 that promote cell wall expansion, CER1 encodes an enzyme of cutin formation, FLA11 encodes a fascilin-type arabinogalactan protein involved in cell adhesion, RTM encodes a mannose-binding lectin, and JAL22 encodes an ER-Golgi transporter that may be involved in the transport of cell wall components to the plasma membrane. The expression of three genes encoding peptidases involved in programmed cell death in xylem, XCP1, XCP2 and the metacaspase-encoding gene $M C 9$ was reduced in $h s r 8-1$ and increased in hsr8-1pft1-2. Similarly the expression of two stress-induced genes, HVA22 and GSTU6 encoding glutathione-S-transferase, was reduced in hsr8-1 compared to Col, and increased in hsr8-1pft1-2 compared to $h s r 8-1$. 


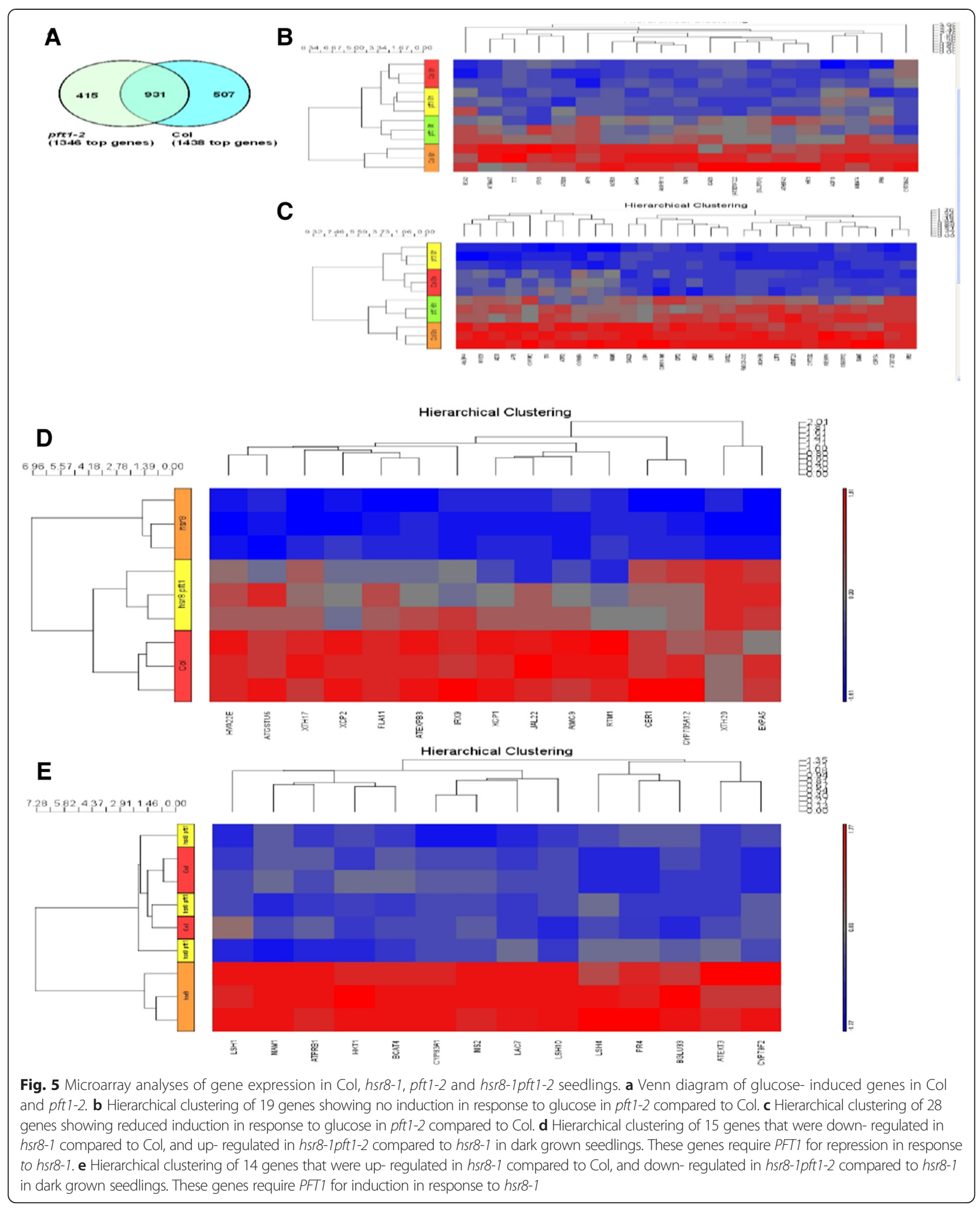


The expression of a diverse set of 14 genes was increased in hsr8-1 compared to $\mathrm{Col}$ and decreased in hsr8-1pft1-2 compared to hsr8-1 (Fig. 5e). These genes required PFT1 for their increased expression in $h s r 8-1$. Three members of the light-dependent short hypocotyl (LHS1, 4 and 10) gene family, encoding conserved nuclear proteins of the ALOG (Arabidopsis LSH1 and Oryza G1) family of transcription factors [38], and five genes encoding enzymes of methionine- and aliphatic glucosinolate biosynthesis [31] required PFT1 for increased expression in hsr8-1. Three genes encoding the cell wall hydroxyproline rich glycoprotein Extensin 3, laccase involved in lignin biosynthesis, and $\beta$-glucosidase 33 also required PFT1 for increased expression in $h s r 8-1$ compared to Col. Finally $H K T 1$, encoding a protein involved in sodium retrieval from xylem, was expressed in a similar pattern.

To extend these analyses, q-RTPCR analyses of genes encoding cell wall components and enzymes with increased expression in hsr8-1 compared to Col [9] was carried out in the double mutant $p f t 1-2 h s r 8-1$. These analyses showed that increased expression in hsr8-1 of EXT3, EXT4, encoding cell wall glycoproteins, and PME17 and PME41, encoding pectin methylesterases, was reduced in hsr8-1pft2-1 (Additional file 1: Figure S4), confirming the increased expression of EXT3 seen in microarray data and extending the range of cell wall-related genes requiring PFT1 in hsr8-1.

\section{MED8 is also required for the expression of selected} genes encoding cell wall components but is a repressor of glucose-induced gene expression

The Mediator complex in Arabidopsis is composed of at least 27 subunits [17], therefore we examined other subunits in addition to PFT1/MED25 for a potential role in sugar- and cell elongation- mediated gene expression. med8 mutants exhibited similar phenotypes to pft1-2 with respect to pathogen responses, flowering time and organ size [39, 40]. Furthermore, the yeast homolog of MED8 was shown to be involved in sugar signalling [41]. To test the involvement of MED8, hsr8-1 was crossed with a loss of function T-DNA insertion med8 mutant, and hypocotyl length in dark developed seedlings was analysed. As shown in Fig. 6a, med8 suppresses the hsr81 short hypocotyl phenotype to the same extend as pft 1 2 (compare with Fi. 1a). We therefore measured expression of the same set of four PFT1-responsive cell wallrelated genes shown in Additional file 1: Figure S4 in hsr8-1 and med8hsr8-1 in dark grown seedlings. Figure $6 \mathrm{~b}$ and $\mathrm{c}$ shows that expression of two of these four, PME17 and PME41, was substantially reduced in med8hsr8-1 compared to hsr8-1. Analysis of glucose- induced gene expression in light- grown med 8 seedlings showed an opposite effect to that observed in the pft1-2
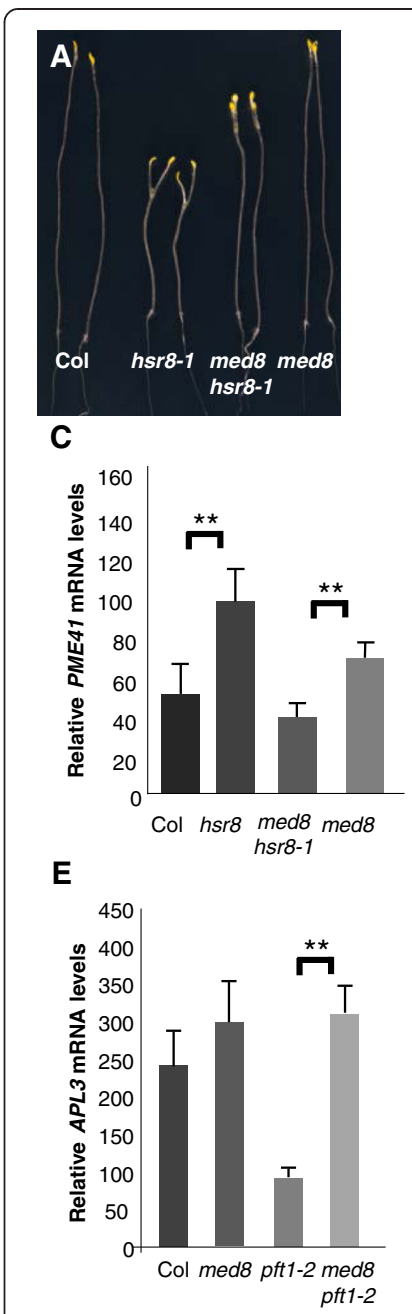
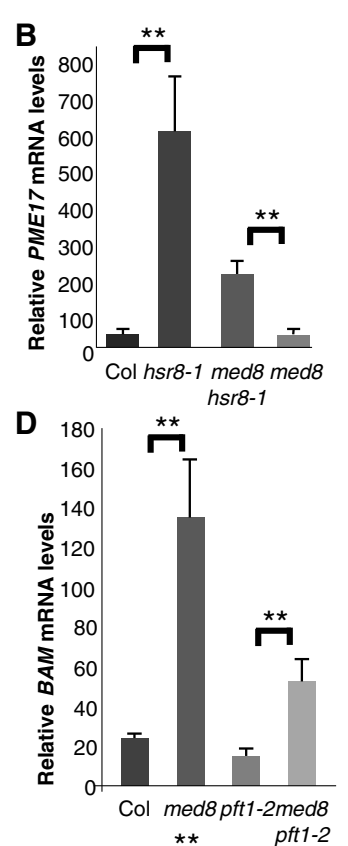

$\mathbf{F}$

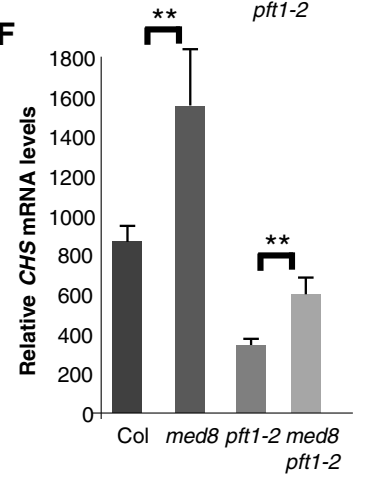

Fig. 6 The MED8 subunit plays a role in sugar responsive growth and gene expression. a Sugar hypersensitive dark development of Col, hsr8-1, med8hsr8-1 and med8 mutants. Seedlings were grown vertically in the dark for 14 days on MS medium containing $1 \%$ Glucose. $\mathbf{b}$ and $\mathbf{c}$ Quantitative Real-time PCR analysis of mRNA levels of cell wall modifying encoding genes PME17 and AtPME41 in Col, hsr8-1, med8hsr8-1 and med8. Seedlings are grown as described in (a) above. Errors bars represent SD from three biological replicates. ${ }^{* *}, p<0.01$ comparing Col to hsr8-1, and med8 hsr8-1 to med8 (Student's t- test). Relative transcript levels (RTL) were calculated relative to the transcript level of the reference gene TUB6 (At5g12250). d to f Quantitative Real-time PCR analysis of BAM, APL3, and CHS mRNA levels in Col, med8, pft1-2 and the double mutant med8pft1-2 in response to glucose. Seedlings were grown on MS medium supplemented with $0.5 \%$ glucose in constant light. After 7 days, the seedlings were transferred to glucose-free liquid MS medium for $24 \mathrm{~h}$ and then treated for $6 \mathrm{~h}$ with $3 \%$ Glucose. Errors bars represent SD from three biological replicates. ${ }^{*}, p<0.01$ comparing Col to med8 and pft1-2 to med8 pft 1-2 (D); ${ }^{* *}, p<0.01$ comparing pft 1-2 to med8 pft1-2 (E); ${ }^{* *}, p<0.01$ comparing $\mathrm{Col}$ to med8 and pft 1-2 to med8 pft 1-2 (F) (Student's t- test). Relative transcript levels (RTL) were calculated using transcript levels of the reference gene TUB6 (At5g12250) 
mutant: the med8 mutant significantly enhances expression of three genes with well-characterised responses to glucose, BAM, APL3 and CHS (Fig. 6d, e and f). This increase was consistently less in the double mutant med8pft1-2 in the analysed genes, suggesting that MED8 and PFT1 have opposing effects on glucose-induced gene expression.

\section{Discussion}

A genetic screen for mutants that suppressed the short hypocotyl phenotype of dark-grown hsr8-1 seedlings identified eight soh mutants. One mutant, soh715hsr8-1, had an intermediate hypocotyl length when grown in the dark (Fig. 1a and b) and also suppressed hypersensitive responses to glucose as assessed by gene expression and anthocyanin accumulation (Fig. 1c and d). The elongated cotyledonary petioles seen in hsr8-1 [9] were also partly suppressed by the soh715 locus (Figs. 1a and 2b), but the main phenotype studied was the large difference in hypocotyl elongation, which was shown to be due to increased cell elongation (Fig. 3a). soh715 was identified as PFT1 (Fig. 2b) [16], encoding a subunit of the Mediator transcription complex [27] and confirmed by the double mutant hsr8-1pft1-2 (Fig. 2c), which was used in subsequent analyses. pft1 mutants exhibit longer hypocotyls in response to phytochrome-mediated signals [16, 42], increasing signalling downstream of PhyA and genetically interact with $H Y 5$ [43]. Figure 4 shows that the dark-development phenotypes of hsr8-1 were not dependent on phyA or PhyB, and hsr8-1 did not significantly influence white- and far- red light responses. Furthermore, the dark development phenotypes of hsr8-1 were not dependent on jasmonate responses [23]. We concluded that PFT1-mediated suppression of reduced hypocotyl elongation in hsr8-1 was not dependent on PFT1 functioning as part of phytochrome- and JA- mediated responses, suggesting PFT1 functions through a independent mechanism(s) to reduce hypocotyl cell elongation during dark development of arabinose-deficient mutants.

The Mediator complex is a functionally conserved regulator of gene expression composed of approximately 30 subunits, forming a complex that docks transcription factors bound to enhancers with core promoter components such as RNA polymerase II [17, 27, 44]. Mediator also has a structural role in chromatin by forming a complex with cohesin that is associated with chromatin looping of promoters [45]. PFT1/MED25 forms part of the tail region of the complex that interacts with transcription factors, while MED8 is part of the head region interacting with core promoter components [27]. In metazoans, many diverse transcriptional regulatory networks converge on Mediator [27], with increasing evidence that different transcription factors interact with different subunits of the tail region. In plants, PFT1/
MED25 and MED8 are required for expression of JAresponsive and fungal resistance genes $[23,46]$ and have antagonistic effects on organ size [40, 47]. PFT1/MED25 is also required for drought-responsive gene expression [42] and is also directly involved in light responses and promoting flowering $[16,43,48]$. The Mediator subunits MED5a/5b repress expression of a set of phenylpropanoid and lignin biosynthetic genes [12, 13], and it was suggested that MED5a/5b may play a direct role in relieving growth repression caused by the phenylpropanoid mutant ref8-1 through a cell wall sensing pathway.

Cluster analyses were conducted to identify two sets of genes in dark grown seedlings that were differentially regulated in hsr8-1 compared to Col and in pft1hsr8-1 compared to $h r 8-1$. These sets comprise genes that required PFT1 for increased or decreased expression in hsr8-1 dark grown seedlings. Of the 15 genes with reduced expression in hsr8-1 compared to Col, and increased expression in hsr8-1pft1-2 compared to hsr8-1, ten encoded proteins involved in cell wall formation (Fig. 6a). Their expression profile shows that the expression of these genes is actively reduced in arabinose- deficient cell walls by PFT1, where they may limit cell wall expansion and/or compensate for altered cell wall composition. Among these are genes for xyloglucan chain modification (XTH17 and XTH20) [36, 49], and XTH17 which has xyloglucan endotransferase- hydrolase activity [50] involved in wall strengthening and expansion in response to shade cues [51,52]. Expression of genes encoding expansins 5 and B3 was also repressed by PFT1 in hsr8-1. These cell wall proteins promote cell wall extensibility, possibly by loosening xyloglucan-cellulose interactions [53].

Fourteen genes encoding regulatory proteins, biosynthetic enzymes and the cell wall protein Extensin $3 \mathrm{had}$ significantly elevated expression in hsr8-1 compared to Col, and reduced expression in hsr8-1pft1-2 compared to hsr8-1 (Fig. 5d). The expression of three genes encoding LSH1, 4 and 10, members of the ALOG family of transcriptional regulators, was coordinately increased in hsr8-1 in a PFT1-dependent pattern. Over-expression of LHS1 led to reduced hypocotyl cell elongation [38], suggesting that PFT1-mediated expression of $L S H$ family members may directly reduce hypocotyl cell elongation in hsr8-1. The increased expression of Extensin 3 and Extensin 4 in hsr8-1 (Additional file 1: Figure S3) was dependent on PFT1, suggesting that the deficient cell walls in hsr8-1 mutants may be strengthened by extensins, and that the reduced expression of Extensin 3 and Extensin 4 in hsr8-1pft1-2 may contribute to increased cell wall extensibility associated with cell elongation.

In Col plants with normal cell walls, PFT1 was required for the increased expression of seven genes encoding proteins that are involved in cell wall extension 
and cell elongation in response to high glucose levels: Expansin 4 encodes a protein that loosens the wall by disrupting hydrogen bonds between cellulose and xyloglucan hemicelluloses [34] and LTP3 and LTP4 encode proteins implicated in cell membrane deposition and cell wall loosening [33]. PIF4 and PIF5 activate LTP3 and Expansin $B 1$ gene expression and promote cell elongation [54], and PhyB negatively regulates this in the light. This is consistent with the known role of PFT1 in PhyB responses $[16,43]$ and suggests PIF4 and PIF5 may function in concert with PFT1 to promote cell elongation in response to light and glucose cues by activating LTP and Expansin gene expression.

Glucose levels strongly influence plant growth, and a key feature of glucose-mediated transcriptional responses involves the rapid coordinated expression of genes encoding enzymes and transporters involved in nutrient acquisition and the synthesis of secondary products and the co-expression of genes involved in ABA responses $[35,55]$. Microarray analyses identified diverse classes of genes whose glucose-induced expression was fully or partly dependent on PFT1/MED25. These genes encoded cell wall- and cell expansion- related proteins, regulatory proteins and enzymes of anthocyanin, flavonoid and glucosinolate biosynthesis, regulators and transporters involved in nutrient uptake, ABA signaling and biosynthetic proteins, and a variety of stress-responsive proteins (Fig. 5b and c). Seven genes encoding enzymes and regulatory proteins in the biosynthesis of glucosinolates [32] required PFT1 for increased expression in response to glucose. In the $h s r 8-1$ mutant PFT1 was also required for the expression of five genes encoding enzymes of glucosinolate synthesis, with MAM1 commonly regulated by glucose. The function of glucosinolate production in hsr8-1 is not known, but the independence of PFT1-mediated hsr8-1 phenotypes on JA indicates that stress responses may not be involved [23]. Notably, glucose-induced expression of $M Y B 75$, encoding a key anthocyanin pathway regulator [28] was completely dependent on PFT1. Recently MED5a and 5b have been shown to repress phenylpropanoid pathway gene expression $[12,13]$, establishing the central role of Mediator in integrating biosynthetic capacity in response to increased carbon supplies. Finally the PFT1- dependent expression of genes encoding nitrate, phosphate and sulphate transporters [56], and the phosphate uptake regulator $S P X 3$, further demonstrate an important coordinating role for PFT1/MED25 in balancing nutrient supplies and carbon availability.

Reduced PFT1 function did not reconstitute wild-type cell wall arabinose content in $h s r 8-1$, as shown by cell wall monosaccharide analyses (Fig. 3b), probably because hsr8-1 is a loss of function allele of MUR4, which encodes the only known enzyme of UDP-arabinose synthesis in Arabidopsis [14]. Only large reductions in cell wall arabinose and fucose led to reduced hypocotyl elongation in the dark [9], which was rescued by low concentrations of borate. Borate cross-links rhamnogalacturonan II and is thought strengthen the cell wall, suggesting changes in cell wall composition and structure lead to reduced elongation in hsr8-1 [9]. Analyses of cell wall polysaccharides using FTIR spectra of cell wall material from dark-developing hypocotyls showed complex quantitative changes in absorbance spectra in hsr8-1 compared to wild-type Col, and in hsr8-1pft1-2 compared to pft1-2 and Col (Fig. 3c and d). Although there were significant differences between genotypes the major component of these differences showed variation across a broad range of wavelengths that precluded identification of specific polysaccharides with altered levels.

\section{Conclusions}

Our analyses demonstrate a central role MED25 and MED8 subunits of the Arabidopsis Mediator complex in transcriptional responses involved in cell elongation, multiple biosynthetic pathways, stress responses, and nutrient acquisition in response to altered carbon availability.

\section{Methods}

\section{Plant material and growth conditions}

All experiments were carried out in the Columbia genetic background. The hsr8-1, hsr3 and hsr4 mutants were isolated as previously described (Li et al. [9]; Baier et al. [57]). The suppressor mutants were isolated from an $h s r 8-1$ fast neutron mutagenized population (seeds were irradiated with 30-40 grays at the HAS KFKI-Atomic Energy Research Institute, Hungary). Plants containing T-DNA insertions in PFT1 (SALK_129555), termed pft1-2, and MED8 (SALK_592406), termed med8, were obtained from The European Arabidopsis Stock Centre (NASC, University of Nottingham, United Kingdom). Seeds were surface sterilized and sown on Murashige and Skoog (MS) medium containing $0.9 \%$ agar and different glucose concentrations. Seeds were then stratified for 3 days at $4{ }^{\circ} \mathrm{C}$ and then grown in continuous light at $22{ }^{\circ} \mathrm{C}$. For dark development experiments, seeds were grown on MS medium containing $1 \%$ glucose, exposed to light for $8 \mathrm{~h}$ and then grown vertically in complete darkness for 2 weeks. For glucose treatment experiments, seedlings were grown on MS medium containing $0.5 \%$ glucose for 7 days and transferred in MS liquid medium without glucose. After $24 \mathrm{~h}$, the medium was changed to MS medium containing $3 \%$ glucose and seedlings were collected $6 \mathrm{~h}$ later. For anthocyanin measurements, seedlings were grown on solid MS medium containing 1 or $3 \%$ glucose for 7 days. 


\section{Genetic screen and cloning of the soh715 mutation}

3200 M2 mutagenized lines were screened individually for increased hypocotyl length, in comparison to hsr8-1, when grown in the dark with $1 \%$ glucose. Potential suppressor mutants were transferred to soil and the M3 progeny was rescreened. The soh715 mutation was mapped by crossing to Landsberg erecta and F2 seeds were screened for long hypocotyls as described above and subsequently genotyped for homozygous hsr8-1 mutation using the LightCycler 480 System and Hybprobes $^{\odot}$ technology (Roche Applied Science and TIB MOLBIOL GmbH). These plants were assumed to be homozygous for the soh715 mutation and used for initial mapping of the mutation. Once the approximate location of the mutation was determined, total RNA of hsr8-1 and soh715hsr8-1 were extracted using the RNeasy plant mini kit (QIAGEN) and used for Affymetrix GeneChip array expression profiling to identify deleted genomic regions (Affymetrix, Santa Clara, CA, USA). We identified a cluster of six consecutive downregulated genes in the soh715hsr8-1-mapped region on chromosome 1 that may be due to a deletion. The soh715hsr8-1 mutant was transformed with genomic fragments containing the sequence of the six genes in the deletion. Additional file 4: Table S3 describes the primers used for cloning genomic fragments using the $\mathrm{TOPO}^{\circ} \mathrm{XL}$ PCR Cloning kit (Invitrogen). Cloned genes were subcloned in the pCAMBIA1300 binary vector using the ApaI restriction site and used for Agrobacterium-mediated transformation. Transgenic T1 plants were screened on $30 \mu \mathrm{g} / \mathrm{mL}$ hygromycin, and complementation of the soh715hsr8-1 long hypocotyl phenotype was assessed in the $\mathrm{T} 2$ generation.

\section{Hypocotyl and anthocyanin measurements}

Hypocotyl length was measured from 14-day old dark grown seedlings $(n=30)$ by scanning plates and using ImageJ software (http://rsb.info.nih.gov/ij/). Anthocyanins were extracted and quantified as described in [57].

\section{Cell wall analysis}

Cell walls were prepared from frozen samples by boiling in $96 \%$ ethanol for 10mins, homogenisation, repeated methanol:chloroform $(2: 3 \mathrm{v} / \mathrm{v})$ extraction, $80 \%$ ethanol extraction and dehydration in $96 \%$ ethanol before drying at room temperature. Aliquots of $50 \mu \mathrm{g}$ were dried, treated with $2 \mathrm{M}$ TFA (trifuoroacetic acid) for $1 \mathrm{~h}$ at $120^{\circ} \mathrm{C}$, and then dried again. Samples were then resuspended in $5 \%(\mathrm{v} / \mathrm{v})$ acetonitrile and injected into an M-Scan High Performance Anion Exchange Chromatography system with Pulsed Amperometric Detection (HPAEC-PAD). Monosaccharides were detected using standards and values expressed as mole \%. Fourier transform Infrared absorbance spectra were collected from
800 to $4000 \mathrm{~cm}^{-1}$ using a Biorad FTS $175 \mathrm{C}$ spectrophotometer. Hypocotyl material was ground and clamped against the diamond element. Two spectra from three biological replicates were obtained. Principle Components Analyses were conducted using Genstat version 15.

\section{Gene expression}

Total RNA was extracted and DNase treated using the RNeasy Plant mini kit (QIAGEN). $2 \mu \mathrm{g}$ were used for reverse transcription (MMLV-RT, Invitrogen) with anchored oligo(dT)23. Quantitative real-time PCR was performed with the LightCycler ${ }^{\circ} 480$ system using the LightCycler 480 SYBR Green I Master 2X (Roche Applied Science) and gene specific primers listed in Additional file 4: Table S3. Primer specificity and efficiency was confirmed by standard and melting curve analyses. Relative transcript levels (RTL) were calculated relative to the transcript level of the reference gene TUB6 (At5g12250) as follows: RTL $=1000 * 2^{- \text {(Cptarget-CpTUB6) }}$. Whole-genome transcriptome analysis was conducted by hybridizing three biological replicate samples of total RNA to Affymetrix GeneChip Arabidopsis ATH1 Genome arrays (Affymetrix, Santa Clara, CA, USA). All steps were conducted at the Nottingham Arabidopsis Stock Centre. Gene expression data were analysed using Partek Genomics Suite 6.6 software (Partek Incorporated, St Louis, USA). The raw CEL files were normalized using the RMA background correction with quantile normalization, log base 2 transformation and mean probe-set summarization with adjustment for GC content. Differentially expressed genes (DEG) were identified by a two-way ANOVA, and $P$-values were adjusted using the FDR (false-discovery rate) method to correct for multiple comparisons. DEG were considered significant if $P$-value was $\leq 0.05$ at a foldchange (FC) of $\geq 2$ with an FDR $<0.05$. Hierarchical clustering in was performed using the default settings in Partek. The average distance between all pairs of objects in the two different clusters was used as the measure of distance between the two clusters, and was measured using Un-weighted Pair-Group Method using arithmetic Averages. Clusters were then merged (agglomerated) until all of the data (genes) were in one cluster.

\section{Availablity of supporting data}

Microarray data are available in the ArrayExpress database (www.ebi.ac.uk/arrayexpress) under accession number E-MTAB-2297.

\section{Additional files}

Additional file 1: Figure S1. Scatter plots of Principle Components 1, 2 and 3 identified from FTIR measurements of cell wall composition. Figure S2. JA responsive genes are not up- regulated in hsr8. Quantitative Real-time PCR analysis of VSP1, VSP2 and ERF1 mRNA 
levels in Col and hsr8. Seedlings were grown vertically in the dark for 14 days on MS medium in the presence of $1 \%$ glucose. Errors bars represent SD from three biological replicates. ${ }^{* *}, p<0.01$ comparing Col to hsr8-1 (Student's t- test). Figure S3. Sugar- regulated gene expression in the single mutant pft 1-2. (A) to (E) Quantitative Real-time PCR analysis of mRNA levels of the glucose-responsive genes APL3, BAM, GBSS1, GPT2, PDC1 in Col and pft 1-2 in response to glucose. Seedlings were grown on MS medium supplemented with $0.5 \%$ glucose in constant light. After 7 days, the seedlings were transferred for $24 \mathrm{~h}$ to glucose-free MS liquid medium (solid bars) and then treated for $6 \mathrm{~h}$ with $3 \%$ glucose (dashed bars). Errors bars represent SD from three biological replicates. Data shown is representative of three independent experiments. ${ }^{*}, p<0.01$ comparing glucose responses in Col to pft1-2 (Student's t- test). (G) and (H) Quantitative Real-time PCR analysis of mRNA levels of the anthocyanin biosynthesis genes CHS, TT6 and FLS in Col and pft 1-2 in response to glucose. Seedlings were grown on MS medium supplemented with $0.5 \%$ glucose in constant light. After 7 days, the seedlings were transferred for $24 \mathrm{~h}$ to glucose-free MS liquid medium (solid bars) and then treated for $6 \mathrm{~h}$ with $3 \%$ glucose (dashed bars). Errors bars represent SD from three biological replicates. Data shown is representative of three independent experiments. **, $p<0.01$ comparing glucose responses in Col to pft1-2 (Student's t- test). Relative transcript levels (RTL) were calculated relative to the transcript level of the reference gene TUB6 (At5g12250). (I) Anthocyanin accumulation in response to glucose in Col and pft 1-2 in response to glucose. Seedlings were grown in continuous light for 7 days on MS containing $1 \%$ glucose (solid bars) or $3 \%$ glucose (dashed bars). Errors bars represent SD from three biological replicates. ${ }^{* *}, p<0.01$ comparing glucose responses in Col to pft1-2 (Student's t- test). Data shown is representative of two independent experiments. Figure S4. PFT1 regulates the expression of genes encoding cell wall- related genes. Quantitative Real-time PCR analysis of genes encoding the pectin methylesterases PME17 (A), PME41 (B), and the extensin proteins AtEXT3 (C) and AtEXT4 (D) in Col, hsr8-1, pft1-2hsr8-1 and pft1-2. Seedlings were grown vertically in the dark for 14 days on MS medium in the presence of $1 \%$ Glucose. Errors bars represent SD from three biological replicates. Panels A-D **, $p<0.01$ comparing Col to hsr8-1 (Student's $t$ - test); panels $B$ and $D^{* *}, p<0.01$ comparing pft 1-2 hsr8-1 to pft 1-2 (Student's $t$ - test). Relative transcript levels (RTL) were calculated using transcript levels of the reference gene TUB6 (At5g12250). (PPTX $97 \mathrm{~kb}$ )

\section{Additional file 2: ANOVA tables of glucose- responsive gene expression in Col and pft1-2. (XLS $1314 \mathrm{~kb}$ ) \\ Additional file 3: ANOVA tables of gene expression in dark grown seedlings of Col, hsr8-1, hsr8-1pft1-2 and pft1-2. (XLS $68 \mathrm{~kb}$ ) \\ Additional file 4: Primers used in gene amplification and Q-RTPCR. (DOCX $100 \mathrm{~kb}$ )}

\section{Abbreviations}

ANOVA: Analysis of variance; ALOG: Arabidopsis LSH1 and oryza G1; BAM: $\beta$-amylase; COR: Cold regulated gene; FTIR: Fourier transform infraRed spectroscopy; GSL: Glucosinolate; JA: Jasmonic Acid; LTP: Lipid Transfer Protein; MED25/PFT1 MEDIATOR25/PHYTOCHROME AND FLOWERING TIME 1; PCA: Principal Component Analysis; PRL1: Pleitropic Regulatory Locus 1; WAK: Cell Wall-Associated Kinase.

\section{Competing interests}

The authors declare they have no competing interests.

\section{Authors' contributions}

MS-A and MWB designed experiments, MS-A, CS, HF and NM conducted experiments, MC-U and SM conducted microarray experiments and analysed data, and MWB and MS-A wrote the manuscript. All authors read and approved the final manuscript.

\section{Acknowledgements}

This work was supported by a Marie Curie Fellowship to M.S-A. (PIEF-GA2009-236779) and BBSRC grants BB/F007582/1 and BB/J004588/1 (GRO) to M.W.B. We thank Dr Thomas Simmons and Professor Paul Dupree of the University of Cambridge for monosaccharide analyses, Dr Charlotte Miller and Dr Rachel Wells of the John Innes Centre for FTIR analyses, Dr Kerry Franklin of the University of Leicester for help with plant growth in different light conditions, and Dr Harry Brumer of the University of British Columbia for providing seeds of an Arabidopsis xth31xth32 double mutant.

\section{Author details}

${ }^{1}$ Cell and Developmental Biology Department, John Innes Centre, Colney Lane, Norwich NR4 7UH, UK. ${ }^{2}$ INRA, Institut Jean-Pierre Bourgin, UMR1318, ERL CNRS 3559, Saclay Plant Sciences, RD10, 78000 Versailles, France. ${ }^{3}$ Nottingham Arabidopsis Stock Centre, University of Nottingham, School of Biosciences, Loughborough LE12 5RD, UK. ${ }^{4}$ Department of Biochemistry, University of Oxford, South Parks Rd, Oxford OX1 3QU, UK.

Received: 10 April 2015 Accepted: 13 August 2015

Published online: 05 September 2015

\section{References}

Somerville C, Bauer S, Brininstool G, Facette M, Hamann T, Milne J, et al.

1. Toward a systems approach to understanding plant cell walls. Science. 2004;306:2206-11.

Reiter W-D. Biosynthesis and properties of the plant cell wall. Curr Opin

2. Plant Biol. 2002;5:536-42.

Geisler DA, Sampathkumar A, Mutwil M, Persson S. Laying down the bricks:

3. logistic aspects of cell wall biosynthesis. Curr Opin Plant Biol. 2008;11:647-52. McFarlane HE, Döring A, Persson S. The cell biology of cellulose synthesis.

4. Annu Rev Plant Biol. 2014;65:69-94 Rolland F, Baena-González E, Sheen J. Sugar sensing and signaling in

5. plants: conserved and novel mechanisms. Annu Rev Plant Biol. 2006;57:675-709.

Sairanen I, Novak O, Pencik A, Ikeda Y, Jones B, Sandberg G, et al. Soluble

6. Carbohydrates Regulate Auxin Biosynthesis via PIF Proteins in Arabidopsis. Plant Cell. 2013;24:4907-16.

Kohorn BD, Kobayashi M, Johansen S, Riese J, Huang L-F, Koch K, et al.

7. An Arabidopsis cell wall-associated kinase required for invertase activity and cell growth. Plant J. 2006;46:307-16.

Hamann T, Bennett M, Mansfield J, Somerville C. Identification of cell-wall

8. stress as a hexose-dependent and osmosensitive regulator of plant responses. Plant J. 2009:57:1015-26.

Li Y, Smith C, Corke F, Zheng L, Merali Z, Ryden P, et al. Signaling from an

9. Altered Cell Wall to the Nucleus Mediates Sugar-Responsive Growth and Development in Arabidopsis thaliana. Plant Cell. 2007;19:2500-15. Koncz C, Dejong F, Villacorta N, Szakonyi D, Koncz Z. The spliceosome-

10. activating complex: molecular mechanisms underlying the function of a pleiotropic regulator. Front Plant Sci. 2012;3:9.

Wolf S, Hématy K, Höfte H. Growth Control and Cell Wall Signaling in Plants.

11. Annu Rev Plant Biol. 2012;63:381-407. Bonawitz ND, Kim Jl, Tobimatsu Y, Ciesielski PN, Anderson NA, Ximenes E,

12. et al. Disruption of Mediator rescues the stunted growth of a lignindeficient Arabidopsis mutant. Nature. 2014;509:1-17.

Bonawitz ND, Soltau WL, Blatchley MR, Powers BL, Hurlock AK, Seals LA,

13. et al. REF4 and RFR1, Subunits of the Transcriptional Coregulatory Complex Mediator, Are Required for Phenylpropanoid Homeostasis in Arabidopsis. J Biol Chem. 2012;287:5434-45.

Burget EG. The Biosynthesis of L-Arabinose in Plants: Molecular Cloning and

14. Characterization of a Golgi-Localized UDP-D-Xylose 4-Epimerase Encoded by the MUR4 Gene of Arabidopsis. Plant Cell. 2003;15:523-31. Mitra RM, Gleason CA, Edwards A, Hadfield J, Downie JA, Oldroyd GED, et al.

15. A Ca2+/calmodulin-dependent protein kinase required for symbiotic nodule development: Gene identification by transcript-based cloning. Proc Natl Acad Sci U S A. 2004;101:4701-5.

Cerdán PD, Chory J. Regulation of flowering time by light quality. Nature.

16. 2003;423:881-5.

Bäckström S, Elfving N, Nilsson R, Wingsle G, Björklund S. Purification of a

17. plant mediator from Arabidopsis thaliana identifies PFT1 as the Med25 subunit. Mol Cell. 2007;26:717-29.

Jiang K, Sorefan K, Deeks MJ, Bevan MW, Hussey PJ, Hetherington AM. The

18. ARP $2 / 3$ complex mediates guard cell actin reorganization and stomatal movement in Arabidopsis. Plant Cell. 2012;24:2031-40. Largo-Gosens A, Hernandez-Altamirano M, Garcia-Calvo L, Alonso-Simon A,

19. Alvarez J, Acebes JL. Fourier transform mid infrared spectroscopy applications for monitoring the structural plasticity of plant cell walls. Front Plant Sci. 2014:5:303. 
Dijkwel PP, Huijser C, Weisbeek PJ, Chua NH, Smeekens SC. Sucrose control

20. of phytochrome A signaling in Arabidopsis. Plant Cell. 1997;9:583-95. Nagatani A, Reed JW, Chory J. Isolation and Initial Chraracterisation of

21. Arabidopsis mutants that are deficient in Phytochrome A. Plant Physiol. 1993;102:269-77. Koornneef M, Rolff E, Spruit CJP. Genetic Control of Light-inhibited

22. Hypocotyl Elongation in Arabidopsis thaliana (L.) Heynh. Z Pflanzenphysiol. 1980;100:147-60.

Kidd BN, Edgar Cl, Kumar KK, Aitken EA, Schenk PM, Manners JM, et al. The

23. mediator complex subunit PFT1 is a key regulator of jasmonate-dependent defense in Arabidopsis. Plant Cell. 2009;21:2237-52.

Ellis C, Turner JG. The Arabidopsis Mutant cev1 Has Constitutively Active

24. Jasmonate and Ethylene Signal Pathways and Enhanced Resistance to Pathogens. Plant Cell. 2001;13:1025-33.

Cano-Delgado A, Penfield S, Smith C, Catley M, Bevan M. Reduced cellulose

25. synthesis invokes lignification and defense responses in Arabidopsis thaliana. Plant J. 2003;34:351-62.

Devoto A, Nieto-Rostro M, Xie D, Ellis C, Harmston R, Patrick E, et al. COl1

26. links jasmonate signalling and fertility to the SCF ubiquitin-ligase complex in Arabidopsis. Plant J. 2002;32:457-66.

Malik S, Roeder RG. The metazoan Mediator co-activator complex as an

27. integrative hub for transcriptional regulation. Nat Rev Genet. 2010;11:761-72. Teng S. Sucrose-Specific Induction of Anthocyanin Biosynthesis in Arabidopsis

28. Requires the MYB75/PAP1 Gene. Plant Physiol. 2005;139:1840-52. Lejay L, Tillard P, Lepetit M, Olive FD, Filleur S, Daniel-Vedele F, et al.

29. Molecular and functional regulation of two NO3- uptake systems by $\mathrm{N}$ - and C-status of Arabidopsis plants. Plant J. 1999;18:509-19.

Koprivova A, Kopriva S. Molecular mechanisms of regulation of sulfate

30. assimilation: first steps on a long road. Front Plant Sci. 2014;5:589. Guo R, Shen W, Qian H, Zhang M, Liu L, Wang Q. Jasmonic acid and

31. glucose synergistically modulate the accumulation of glucosinolates in Arabidopsis thaliana. J Exp Bot. 2013;64:5707-19.

Gigolashvili T, Yatusevich R, Berger B, Müller C, Flügge U-I. The R2R3-MYB

32. transcription factor HAG1/MYB28 is a regulator of methionine-derived glucosinolate biosynthesis in Arabidopsis thaliana. Plant J. 2007;51:247-61. Nieuwland J, Feron R, Huisman BAH, Fasolino A, Hilbers CW, Derksen J, et al.

33. Lipid transfer proteins enhance cell wall extension in tobacco. Plant Cell. 2005;17:2009-19

Goh H-H, Sloan J, Dorca-Fornell C, Fleming A. Inducible repression of

34. multiple expansin genes leads to growth suppression during leaf development. Plant Physiol. 2012:159:1759-70.

Li Y. Establishing glucose- and ABA-regulated transcription networks in

35. Arabidopsis by microarray analysis and promoter classification using a Relevance Vector Machine. Genome Res. 2006;16:414-27.

Eklöf JM, Brumer $\mathrm{H}$. The XTH gene family: an update on enzyme structure,

36. function, and phylogeny in xyloglucan remodeling. Plant Physiol. 2010;153:456-66.

Wu AM, Hornblad E, Voxeur A, Gerber L, Rihouey C, Lerouge P, et al.

37. Analysis of the Arabidopsis IRX9/IRX9-L and IRX14/IRX14-L Pairs of Glycosyltransferase Genes Reveals Critical Contributions to Biosynthesis of the Hemicellulose Glucuronoxylan. Plant Physiol. 2010;153:542-54. Zhao L, Nakazawa M, Takase T, Manabe K. Overexpression of LSH1, a

38. member of an uncharacterised gene family, causes enhanced light regulation of seedling development. Plant J. 2004;37:694-706. Kidd BN, Cahill DM, Manners JM, Schenk PM, Kazan K. Diverse roles of the

39. Mediator complex in plants. Semin Cell Dev Biol. 2011;22:741-8. $X u R$, Li Y. The Mediator complex subunit 8 regulates organ size in

40. Arabidopsis thaliana. Plant Signal Behav. 2012;7:182-3. la Cera de T, Herrero P, Moreno-Herrero F, Chaves RS, Moreno F. Mediator

41. Factor Med8p Interacts with the Hexokinase 2: Implication in the Glucose Signalling Pathway of Saccharomyces cerevisiae. J Mol Biol. 2002;319:703-14. Elfving N, Davoine C, Benlloch R, Blomberg J, Brännström K, Müller D, et al.

42. The Arabidopsis thaliana Med 25 mediator subunit integrates environmental cues to control plant development. Proc Natl Acad Sci U S A. 2011;108:8245-50.

Klose C, Buche C, Fernandez AP, Schafer E, Zwick E, Kretsch T. The Mediator

43. Complex Subunit PFT1 Interferes with COP1 and HY5 in the Regulation of Arabidopsis Light Signaling. Plant Physiol. 2012;160:289-307. Conaway RC, Conaway JW. Function and regulation of the Mediator

44. complex. Curr Opin Genet Dev. 2011;21:225-30.
Kagey MH, Newman JJ, Bilodeau S, Zhan Y, Orlando DA, van Berkum NL,

45. et al. Mediator and cohesin connect gene expression and chromatin architecture. Nature. 2010;467:430-5.

Ou B, Yin K-Q, Liu S-N, Yang Y, Gu T, Wing Hui JM, et al. A high-throughput

46. screening system for Arabidopsis transcription factors and its application to Med25-dependent transcriptional regulation. Mol Plant. 2011;4:546-55. Xu R, Li Y. Control of final organ size by Mediator complex subunit 25 in

47. Arabidopsis thaliana. Development. 2011;138:4545-54. Iñigo S, Alvarez MJ, Strasser B, Califano A, Cerdán PD. PFT1, the MED25

48. subunit of the plant Mediator complex, promotes flowering through CONSTANS dependent and independent mechanisms in Arabidopsis. Plant J. 2012;69:601-12.

Kaewthai N, Gendre D, Eklof JM, Ibatullin FM, Ezcurra I, Bhalerao RP, et al.

49. Group III-A XTH Genes of Arabidopsis Encode Predominant Xyloglucan Endohydrolases That Are Dispensable for Normal Growth. Plant Physiol. 2012;161:440-54

Zhu XF, Wan JX, Sun Y, Shi YZ, Braam J, Li GX, et al. Xyloglucan

50. Endotransglucosylase-Hydrolase17 Interacts with Xyloglucan Endotransglucosylase-Hydrolase31 to Confer Xyloglucan Endotransglucosylase Action and Affect Aluminum Sensitivity in Arabidopsis. Plant Physiol. 2014;165:1566-74.

Miedes E, Suslov D, Vandenbussche F, Kenobi K, Ivakov A, Van Der Straeten D,

51. et al. Xyloglucan endotransglucosylase/hydrolase (XTH) overexpression affects growth and cell wall mechanics in etiolated Arabidopsis hypocotyls. J Exp Bot. 2013;64:2481-97.

Sasidharan R, Chinnappa CC, Staal M, Elzenga JTM, Yokoyama R, Nishitani K,

52. et al. Light Quality-Mediated Petiole Elongation in Arabidopsis during Shade Avoidance Involves Cell Wall Modification by Xyloglucan Endotransglucosylase/ Hydrolases. Plant Physiol. 2010;154:978-90.

Cosgrove DJ. Loosening of plant cell walls by expansins. Nature.

53. 2000:407:321-6. de Lucas M, Davière J-M, Rodríguez-Falcón M, Pontin M, Iglesias-Pedraz JM,

54. Lorrain $\mathrm{S}$, et al. A molecular framework for light and gibberellin control of cell elongation. Nature. 2008;451:480-4.

Baena-González E, Sheen J. Convergent energy and stress signaling. Trends

55. Plant Sci. 2008;13:474-82.

Koprivova A, Calderwood A, Lee B-R, Kopriva S. Do PFT1 and HY5 interact in

56. regulation of sulfate assimilation by light in Arabidopsis? FEBS Lett. 2014:588:1116-21.

Baier M, Hemmann G, Holman R, Corke F, Card R, Smith C, et al.

57. Characterization of mutants in Arabidopsis showing increased sugar-specific gene expression, growth, and developmental responses. Plant Physiol. 2004;134:81-91.

\section{Submit your next manuscript to BioMed Central and take full advantage of:}

- Convenient online submission

- Thorough peer review

- No space constraints or color figure charges

- Immediate publication on acceptance

- Inclusion in PubMed, CAS, Scopus and Google Scholar

- Research which is freely available for redistribution 